\title{
Formation Mechanism and Clinical Application of the Dominant Eye
}

\author{
Qiu Liping ${ }^{1}$, Wang Hua ${ }^{2}$ \\ ${ }^{1}$ Department of Medical College, Hunan Provincial People's Hospital, The First Affiliated Hospital of Hunan Normal University, Changsha, \\ China \\ ${ }^{2}$ Center for Ophthalmic Optics, Hunan Provincial People's Hospital, Changsha, China
}

Email address:

463557469@qq.com (Qiu Liping), wanghuaeye@163.com (Wang Hua)

\section{To cite this article:}

Qiu Liping, Wang Hua. Formation Mechanism and Clinical Application of the Dominant Eye. International Journal of Ophthalmology \& Visual Science. Vol. 5, No. 2, 2020, pp. 47-52. doi: 10.11648/j.ijovs.20200502.12

Received: March 12, 2020; Accepted: March 26, 2020; Published: April 7, 2020

\begin{abstract}
Eye dominance is often defined as a preference for the visual input of one eye to the other. The dominant eye is a common human side of the functional advantages and is significant for clinical diagnosis and treatment of eye disease. While significant research has been performed on the dominant eye, researchers are still unable to come to a general consensus because they are unable to delve deeply into researching the dominant eye in more details. This is very much unlike the study of the human limbs as, over the years, scientists have been able to successfully research its functionality in every aspect. Research from the dominant eye is divided into three phases: Pre-critical, critical period, after the critical period. Ocular dominance is the advantage of a three-dimensional situation, including sighting, movement, acuity, representing different aspects respectively. There are different advantage aspects with different inspection methods. The dominant eye in oblique amblyopia treatment, optometry, refractive surgery has an important application value. With the rapid development of marginal disciplines such as brain science, neurodevelopment, and neurobiology, the rapid advancement and widespread application of neuroelectrophysiology and optical imaging technology have led to deeper research and understanding of the dominant eyes. The purpose of this article is to assess the focus on the dominant eye formation mechanism, inspection methods and review the status of clinical diagnosis and the treatment of eye diseases.
\end{abstract}

Keywords: Dominant Eye, Formation Mechanism, Dominant Eye Measurement

\section{Introduction}

Humans have pairs of organs that have the same function and are symmetrically distributed, but this symmetry is not absolute. Some common examples are the dominant eye, handedness, foot preference, the advantages of the brain etc. Since Porta first proposed the concept of the dominant eye in 1593 and divided the dominant eye into right eye advantage and left eye advantage, researchers' studies of the dominant eyes have never stopped. Unlike the study of the human limbs, which has progressed exponentially over the years, investigative research for the dominant eye still continues while researchers fail to reach a consensus [1]. In 1903, Rosenbach [2] first discussed the problem of eye superiority. He believed that although both eyes received the same or different visual signals, most people had one dominant eye, but the superior eye was not necessarily the one with the better vision. Clinically, the eye with positioning priority is generally understood to be the dominant eye. The actual advantages include the following aspects: sighting, movement, acuity, and the competitive relationship representing different levels respectively [3]. The eye that plays a dominant role in positioning is called the sighting dominant eye. In the wide movement, that is, during the process of increasing the viewing angle of both eyes, the eye that always maintains the attention is called the sports superiority eye [4]. The perceptual advantage eye mainly reflects the lateral advantage of the cerebral cortex in processing binocular visual input information. When complex cells in the visual cortex receive binocular stimulation input, one eye tends to dominate, and the dominant eye produces more frequent discharges than the non-dominant eye [5]. This reflects the relatively sensitive eye of the central brain to binocular visual signals, known as 
the sensory dominant eye. The sensory dominant eye formation mechanism is similar to the binocular competition inhibition mechanism in patients with strabismus and amblyopia [6]. Previous studies have suggested that the dominant position of the dominant eye is formed during the plastic phase of visual development, and a dominant column is formed in the brain. It is difficult to change without strong stimulation [7-8].

\section{Formation Mechanism of the Dominant Eye}

For binocular vision mammals, external objects are imaged on the retina through a refractive medium, and the retinal image is projected along the optic nerve and through the lateral geniculate body to the cerebral cortex. In this process, the axons retained the original nerve layers of different eyes, and eventually formed cortical cell plaques of different eyes in the cortex. Huber found through in vitro microelectrodes [9] that these cortical cells have the same orientation to the axis gathered together, spaced apart from each other to form a vertical columnar structure. Cortical cell columns of different orientations are driven by the left and right dominant eyes, respectively. Such dominant cortical cell columns are called eye dominant columns. The development of the eye superiority column is divided into three phases, before the critical period, the critical period, and after the critical period. The ocular dominant column is formed before the critical period [9], which is the embryo stage. Developmental impairment in the visual system during the critical period can cause eye disease, such as Infantile Nystagmus [10]. Crowley [8], and other studies, found that monocular or binocular visual deprivation before the critical period did not affect the formation of the dominant eye column. It may be argued that the self-generation activity of the Retinal and Lateral Geniculate Body (LGB) plays an important role in the development of the dominant column of the eye, which may be related to the spontaneous discharge of the retinal and brain cells. Studies have shown [9] that using drugs to block spontaneous discharge of the retina before the formation of the dominant column, and normal separation of the dominant column has not been observed in adult mice. The above proves that blocking the autogenous activity of the early retina will affect the dominant column of the cerebral cortex formation. The critical period is the age range from birth to 3 years, the period during which human nerves gradually mature. The visual system can adjust and change neural connections and synaptic structures according to the stimulation of the visual environment. After the critical period, the dominant eye and dominant column stabilize. Without greater stimulation, the plasticity is small. Some recent studies have used different methods, such as using VEP to record activity-dependent signals and the rapid expression of immediate early genes (lEos) that mark cortical cells, which showed evidence that, in adult mice, the dominant eye changes in the dominant column. These experiments indicate the presence of plasticity in the dominant eye after the critical period in mice [11-12]. Latest research, compared contrast thresholds between the dominant and non-dominant eyes, have found that the dominant eye was more sensitive overall than the non-dominant eye to both stimuli [13].

\section{Dominant Eye Measurement}

Dominant eye is a three-dimensional advantage structure composed of sighting, movement, and binocular sensory competition. The eye advantage itself is multi-faceted, and clinical methods for measuring dominant eye are different according to different aspects of the dominant state. All examination methods must be performed with the correction of Refractive error.

\subsection{Sighting Dominant Eye Measurement}

Sighting dominant eyes can be measured using the hole-in-card method, pointing-a-finger method, and prism method.

Hole-in-Card Method: The subject was given a piece of cardboard in which there was a central circular hole $3 \mathrm{~cm}$ in diameter. The patient was asked to hold the cardboard with both hands and to view a target $6 \mathrm{~m}$ away through the hole, with both eyes open. When the dominant eye was covered, the target could not be seen through the hole. Alternatively, when the nondominant eye was covered, the dominant eye continued to see the target through the hole.

Pointing-a-Finger Method: Gaze at a certain point in the distance, put your thumb on the line of sight. Ask the patients to close his right eye, and if his thumb covers the point, it is the left dominant eye. Otherwise, the right eye is the sighting dominant eye. This inspection process requires that the patient not move. Pointing-a-Finger does not require an inspection device, but it is difficult to understand, and some patients treat one hand as two because of physiological diplopia, which makes inspection difficult.

Prism Method: The basal-down prism is placed in front of the eyes, and both eyes are quickly covered to determine the superiority of attention in the eyes [14].

Studies have shown [15] that the results of hole-in-card method and pointing-a-finger method for determining the dominant eyes are equivalent. Because the card hole method is simple and easy to understand, it is widely used in clinical measurements of sighting dominant eye.

\subsection{Motor Dominant Eye Measurement}

Motor dominant eyes can be evaluated using convergence near-point test and the Dunlop method.

The convergence near-point test: Ocular dominance was confirmed by having the subject fixate on an object that was moved toward the nose until divergence occurred in one eye, which was taken to be the nondominant eye. The divergence in one eye was judged by an ophthalmologist.

The Dunlop Method: Determining the dominant eye using Synoptophore is based on binocular accommodation. It is 
taking the subjective feeling of the examinee as the end point of the examination, so this method is difficult to understand. Therefore, the convergence near-point test is widely used as the examination method to measure the motor dominant eye.

\subsection{Sensory Dominant Eye Measurement}

The methods that can be used for the sensory dominant eye include the additional lens method, the Worth four-point method, and the continuous flicker suppression method.

Additional Lens Method: Add negative lenses of the same degree in front of both eyes. If the lens has worse visual quality when added to the right eye, the right eye is the sensory dominant eye, otherwise the left eye is the sensory dominant eye. The principle of this method is that reducing the monocular vision, the visual quality of binocular retinal imaging is affected, and the sensory dominant eye blur has a greater impact on the binocular retinal imaging quality [16-20].

Worth Four-Point Method: The examinee wears lenses with red eyes on the right and green on the left. Looking at the four-point light on the comprehensive optometry device, if the patient sees four lights, two red lights and two green lights, the right eye is the perceptual dominant eye, and if three green lights and one red light, the left eye is perceptual Dominant eye. Studies have shown that [21] Worth's four-point method has its test results disturbed by the subjects' perception, understanding, and color. At the same time, the inhibition reaction, diplopia reaction, and fusion reaction can also affect the results.

The continuous flicker suppression method [22] uses a computerized system based on the principle of "two-eye competitive balance" to implement a precise judgment and quantify the size of the dominant superior eye.

The above three inspection principles are based on the normal sensory fusion of the two eyes to determine which eye of the retinal image is dominant during the fusion process of the two eyes, thereby determining the sensory dominant eye.

\section{The Status and New Research Progress of the Dominant Eye}

\subsection{Feature Distribution of the Dominant Eye}

\subsubsection{Dominant Eyes and Right or left eye}

Studies have shown [1, 23-26] that, whether at home or abroad, the distribution ratio of right eye dominance in the population is higher than that of the left eye. Linke et al.'s [27] study of 10,264 patients with myopia showed that the right dominant eye is higher in men $(64.9 \%)$ than in women $(62.4 \%)$.

\subsubsection{Dominant Eyes and Development of Myopia}

The prevalence of myopia has increased dramatically in recent years in many parts of the world [28]. Nevertheless, the etiology of myopia remains unclear, although both genetic and environmental components are thought to be involved. A retrospective study of 2,453 people by Eser et al. [29] showed that there was no significant difference between the average equivalent spherical lens of the dominant and non-dominant eyes. Yang et al.'s [30] research showing that dominant eyes have no major significance for the development of myopia. However, with the deepening of the understanding of dominant eyes, Cheng et al. [31] first pointed out that dominant eyes were more myopic than non-dominant eyes in 55 adult cases with Anisometropia > $0.5 \mathrm{D}$. It is statistically significant, and it is proposed, that different people have different degrees of advantages in their superior eyes. The greater the degree of superiority, the more likely they are to develop a high degree of refractive error. The current view of retinal defocusing to explain the development of myopia is supported by relevant researchers [32]. A recent study [33-35] showed that if the accuracy of the adjustment in near work fails to maintain a defocused retinal image, it can lead to the development of myopia. The dominant eye plays a primary role in accommodation during binocular viewing, with an increased static tonus of its ciliary muscle compared with that of the nondominant eye $[8,36]$. The increased static tonus of the ciliary muscle may interfere with normal accommodative responses. Because ocular dominance represents the tendency to prefer visual input from the dominant eye, the preferential use of the dominant eye for viewing might render the dominant eye more myopic than the nondominant eye.

However, Linke et al. [27], Ito et al. [37] and Wang et al. [38] have shown that non-dominant eye refractive power is higher, especially for people of high Anisometropia. People with Anisometropia have a higher probability of experiencing non-dominant eye refraction errors. When the Anisometropia is greater than $2.5 \mathrm{D}$, the refractive power of non-dominant eye is high and statistically significant. So, does the non-dominant eye's myopia and myopic astigmatism develop faster? Or, is it because the myopia and high myopia astigmatism have a tendency to develop into non-dominant eyes? Due to the limitation of cross-sectional design, these studies have not been concluded.

\subsubsection{Dominant Eyes and Dominant Hands}

Studies [39] indicate that the presence of the dominant hand will affect the measurement results of the dominant eye. $\mathrm{Xia}$ et al. [40] considered that the formation of myopia is closely related to the formation of right-handedness. The reason is that the width of the occipital cortex visual center, left occipital region width ratio of non-right-handers than right-hand were large, which may cause the right limb to receive more nerve, more turn right limb movement also stimulates the left side of the brain's development, the formation of childhood handedness leading to the left and right brain's visual cortex development imbalance, thereby forming a side of the visual function. The study of the card hole method and the radio spot approach method [41] shows that the former has higher right-eye dominance than the latter, because right-handedness is a more common trait, and the close relationship between eyes and hands is gradually 
established. If the eye dominant is not obvious, it will appear to the right eye dominance.

\subsection{Dominant Eye and Optometry}

Studies have shown that [42] an optician should maintain the same dominant eye before and after correcting refractive error. This is because the dominant eye column of the occipital visual cortex of the human brain is formed before the critical period, it is difficult to change after the dominant column is formed, but the vision of the dominant eye is reduced for a long time. The input of ocular visual information causes the dominant eye to drift to clear vision. As a result, the dominant eye is remolded. Increasing the burden on the non-dominant ciliary muscles during this process can cause subjective discomfort to the wearer [43].

\subsection{Dominant Eye and Oblique Amblyopia}

The study found that for patients with intermittent exotropia, the dominant eye with small-angle intermittent exotropia can be determined by measuring the Sighting dominant eye, and the coincidence rate between the Sighting dominant eye and the dominant eye is as high as $97 \%$ [44]. Correcting common strabismus in the dominant eye has the advantages of large correction, less surgical muscles involved, quick recovery of binocular monoopia, and improvement of amblyopia. This view has been recognized by many scholars both at home and abroad [45].

In the treatment of amblyopia, the dominant eye is covered, and appropriate visual stimulation is given to the amblyopic eye to slow down or eliminate the long-term suppression of the amblyopic eye from the dominant eye, as a result, the vision of the amblyopic eye improves. This is based on the plasticity principle of the dominant eye. By suppressing and covering the dominant eye, after the sensitive period of amblyopia correction, the changes in the dominant cortical column of the cerebral cortex stabilize, and finally, the vision balance of both eyes is achieved.

\subsection{Dominant Eye and Refractive Surgery}

With the improvement of people's material life, the requirements for visual quality is also increasing. Currently, only good eyesight cannot meet the needs of patients and it is also no longer the only goal of refractive surgery. Refractive doctors are mostly concerned about how to improve the visual quality of patients and improve patient satisfaction after surgery. A clinical study found that some patients with visual acuity improved significantly in their dominant eye. However, most often complained about the decline in the quality of visual comfort after surgery. The current analysis of the decline in satisfaction after refractive surgery mainly lies in the occurrence of intraoperative and postoperative complications. Such as, decentration and postoperative decreased tear film quality, less attention is paid to the conversion of dominant eyes before and after refractive surgery. In a research of 118 patients who had undergone LASIK eye surgery, it was discovered that the dominant eye did not convert within the 3 months limitation period. Luo et al. [46] found that the patients could occur dominant eye conversion after LASIK surgery and pointed out that it may be related to preoperative uncorrected visual acuity level and the degree of myopia. In his study, Wang et al. [47] noted that LASIK surgery worked as a stimulant which causes a conversion in the dominant eye while correcting the myopia. This was proof that ocular dominance plasticity is present in the dominant eye after the critical period. Patients who have Anisometropia with dominant eye, as well as a high degree of myopia of both eyes, tend to change the dominant eye after LASIK surgery. Studies have shown that refractive surgery will more than likely affect the dominant eye choice after breaking the original balance of binocular vision in the binocular dominant eye then for the implementation of myopic surgery. Why convert the dominant eye of some patients, For those patients who have a dominant eye conversion after surgery, will they switch back to the establishment of binocular vision balance? There is no report of the research.

$\mathrm{Hu}$ et al. [48] researched the excimer laser treatment of presbyopia surgery design monocular as the basis, using a selective inhibition of the brain, the dominant eye is completely corrected for farsightedness, while the non-dominant eye retains a certain degree of nearsightedness for nearsightedness. Eyes monovision obtained distance vision after surgery was significantly better than the dominant eye for distance vision. When the human eye near vision by the ciliary muscle contraction to cause a change of curvature so that the lens near object can be clearly imaged on the retina, ciliary muscles relax so that distant objects are in clear view of the human eye object [49]. The presbyopia becomes blurred as a result of age-related decline and ones' ability to regulate ciliary body [49-50]. Clinically, based on the theory of monocular vision, presbyopic patients can see both near and far objects by wearing contact lenses, or by obtaining corneal refractive surgery or intraocular refractive surgery [51-52]. Some studies [53-54] show that in the treatment of presbyopia, "monovision" has been widely used and accepted in clinical practice. The success rate of contact lens treatment for presbyopia is 70\% - 80\% [54-57]. Clinical studies have shown that [58] monovision clear view both near and distance objects, design implementation monovision for early presbyopia patients will have good efficacy.

\section{Conclusions}

The dominant eye is one of the advantages of the common functional characteristics of human beings. Researchers have made great progress in the research of the dominant eyes; however, mankind still has not solved the mystery. Whether or not it is dependent on strabismus, amblyopia treatment, or by fitting glasses, excimer laser surgery, or intraocular lens implant surgery treatment of refractive errors, all those diseases need to take the importance of the dominant eye into account. 


\section{References}

[1] Pointer J S. Sighting dominance, handedness, and visual acuity preference: three mutually exclusive modalities? [J]. Ophthalmic and Physiological Optics, 2001, 21 (2): 117-126.

[2] Guntram Kommerell, Christina Schmitt, Miriam Kromeier, Michael Bach. Ocular prevalence versus ocular dominance [J]. Vision Research, 2003, 43 (12)

[3] Jiang Feng, Huang YiFei, Zhang Bin. Dominant Eye Typing and Clinical Research Progress [J]. Chinese Journal of Optometry and Visual Science, 2015, 17 (6): 381

[4] Reiss MR. Ocular dominance: some family data. Laterality. 1997; 2: 7-16

[5] Yu Lu, Zhao kanXing. A Study on the Dominant Column and Its Plasticity in Visual Development [J]. International Review of Ophthalmology, 2007, 31 (06): 403-406.

[6] Sengpiel F, Blakemore C, Kind PC, Harrad R. Interocular suppression in the visual cortex of strabismic cats. J Neurosci

[7] Sengpiel F, Kind P C. The role of activity in development of the visual system [J]. Curr Bio, 2002, 12 (23) 818

[8] Crowley J C, Katz L C. Ocular dominance development revisited [J]. Cur Opin In Neurobiol, 2002, 12 (1) 104

[9] Huberman A D, Speer C M, Chapman B. Spontaneous Retinal Activity Mediates Development of Ocular Dominance Columns and Binocular Receptive Fields in V1 [J]. Neuron, 2006, 52 (2): 247-254.

[10] Foutch BK. The Dominant Eye: Dominant for Parvo-But Not for Magno-Biased Stimuli? Vision (Basel) 2020; 4: DOI: 10.3390/vision4010019.

[11] Hofer SB, Mrsic-Flogel TD, Bonhoeffer T, Hubener M. Prior experience enhances plasticity in adult visual cortex. Nat Neurosci. 2006 Jan, 9 (1): 127-132.

[12] Lickey ME, Pham TA, Gordon B Swept contrast visual evoked potentials and their plasticity following monocular deprivation in mice. Vision Res. 2004, 44 (28) 3381-7

[13] Moshkovitz A, Lev M. Monocular and Binocular Temporal Visual Perception of Infantile Nystagmus. Sci Rep 2020; 10: 4946. DOI: $10.1038 / \mathrm{s} 41598-020-61914-2$.

[14] Melissa L. Rice, David A. Leske, Christina E. Smestad, Jonathan M. Holmes. Results of ocular dominance testing depend on assessment method [J]. Journal of AAPOS, 2008, $12(4)$.

[15] Li KeJia, Liu Han Qiang. Comparison of three different methods for examination of dominant eye [J]. International Journal of Ophthalmology, 2009, 9 (03): 502-503.

[16] Ukai K. Ando H. Kuze Binocular rivalry alternation rate declines with age Percept Motor Skills 2003: 97: 393-397

[17] Handda T, Uozato H, Higa R, et al Quantitative measurement of ocular dominance using binocular rivalry induced by retinometer. J Cataract Refract Surg 2006; 32: 831-836.

[18] Heinrich SP, Kromeier M. Bach M. Kommerell G. Vernier acuity for stereodisparate objects and ocular prevalence. Vis Res 2005: 45: 1321-1328
[19] Lopes-Ferreira D, Neves H. Queiros A. et al. Ocular dominance and visual function testing [J]. Biomed Res Int. 2013: 238943

[20] Yang E. Blake R, McDonald JE. A new interocular suppression technique for measuring sensory eye dominance, Invest Ophthalmol Vis Sci, 2010, 51 (I): 588-93

[21] Qiu KaiKai, Zhang ShaoBin, Zhou ZhongXia. Comparison of the measurement results of dominant eyes with worth fourpoint method and hole-card method under the condition of refraction habit correction and full correction [J]. Chinese Journal of Optometry \& Ophthalmology, 2006 (01): 36-38.

[22] Kamis U, Gunduz K, Okudan N, et al. Relationship between Eye Dominance and Pattern Electroretinograms in Normal Human Subjects [J]. International Journal of Neuroscience, 2005, 115 (2): 185-192.

[23] Dane S, GüMüSTEKIN, KENAN. CORRELATION BETWEEN HAND PREFERENCE AND DISTANCE OF FOCUSING POINTS OF TWO EYES IN THE HORIZONTAL PLANE [J]. International Journal of Neuroscience, 2002, 112 (10): 1141-1147.

[24] Zheng Lianbin, ZhouDan. A Study on the Characteristics of Seven Asymmetric Behaviors of the Han Nationality in Tianjin [J]. Journal of Tianjin Normal University (Natural Science Edition), 2003 (04): 19-22.

[25] Zheng LianBin, Li Shuyuan, Han ZaiZhu, Lu ShunHua. Human asymmetric behavior characteristics [J]. Journal of Tianjin Normal University (Natural Science Edition), 2001 (03): 58-61.

[26] Yu YueSheng, Cao MingXian, Wang keSong, Jie JuQuan, Luo ZaiGang, Li ZhongYan. A Study on the Characteristics of Asymmetric Behavior of Eight Ethnic Groups in Guizhou [J]. Guizhou Medicine, 2004 (04): 373-376.

[27] Linke SJ, Baviera J, Munzer G, Steinberg J, Richard G, Katz T. Association between ocular dominance and spherical/astigmatic anisometropia, age, and sex: analysis of 10, 264 myopic individuals. Invest Ophthalmol Vis Sci. 2011; 52: 9166-9173.

[28] Saw SM, Katz J, Schein OD, et al. Epidemiology of myopia. Epidemiol Rev. 1996; 18: 175-187.

[29] Eser I, Durrie DS, Schwendeman F, Stahl JE. Association between ocular dominance and refraction. J Refract Surg. 2008; 24: 685-689.

[30] Yang Z, Lan W, Liu W, et al. Association of ocular dominance and myopia development: a 2-year longitudinal study. Invest Ophthalmol Vis Sci. 2008; 49: 4779-4783.

[31] Cheng CY, Yen MY, Lin HY, Hsia WW, Hsu WM. Association of ocular dominance and anisometropic myopia. Invest Ophthalmol Vis Sci. 2004; 45: 2856-2860.

[32] Goss DA, Wickham MG. Retinal-image mediated ocular growth as a mechanism for juvenile onset myopia and for emmetropization: a literature review. Doc Ophthalmol. 1995; 90: 341-375.

[33] Mapp AP, Ono H Barbeito R. What does the dominant eye dominate? A brief and somewhat contentious review. Percept Psychophys. 2003: 65: 310-317.

[34] Porac C. The dominant eye. Psychol Bull. 1976: 83: 880-897 
[35] Le Vay S. Stryker MP, sha-tz CJ Ocular dominance columns and their development in layer IV of the cat's visual cortex: a quantitative study. J Comp Neurol. 1978.179: 223-244

[36] Ibi K. Characteristics of dynamic accommodation responses: comparison between the dominant and nondominant eyes. Ophthalmic Physiol Opt. 1997; 17: 44-54.

[37] Ito M, Shimizu K, Kawamorita T, et al. Association Between Ocular Dominance and Refractive Asymmetry [J]. Journal of refractive surgery (Thorofare, N. J.: 1995), 2013, 29 (10): 1-5.

[38] Wang Q, Wu Y, Liu W, Gao L. Dominant Eye and Visual Evoked Potential of Patients with Myopic Anisometropia. Biomed Res Int 2016; 2016: 5064892.

[39] Nimet nay Gundoan. Two methods to study the relationship between the dominant eye and the left / right hand of college students (English) [J]. International Journal of Ophthalmology, 2009, 9 (12): 2273-2277.

[40] Li Xia, Gao XiaoWei, Lei Ying. Clinical observation on the relationship between adolescent myopia and right-handedness [J]. International Journal of Ophthalmology, 2009, 9 (10): 2011-2012.

[41] Zhu ReYuan, Qu XiaoMei. The meaning and implementation of medical optometry [J]. Chinese Journal of Optometry \& Ophthalmology, 2002 (02): 116-117.

[42] John T. Flynn, Zhou YiDing. Revisiting amblyopia [J]. Foreign Medicine. Ophthalmology, 1992 (06): 361-365.

[43] Quah BL, Lu Y, Smith D. The association of ocular dominance and the preferred eye for fixation in intermittent exotropia: a guide to choosing the eye for unilateral surgery. Am Orthopt J 2001; 51: 111-115

[44] Jin Wook Jeoung, Min Joung Lee, Jeong-Min Hwang. Bilateral Lateral Rectus Recession Versus Unilateral Recess-Resect Procedure for Exotropia With a Dominant Eye [J]. American Journal of Ophthalmology, 2005, 141 (4).

[45] Li KeJi, LiuHanQiang. Study of ocular dominance's changes in myopic patient after LASIK [J]. International Journal of Ophthalmology, 2009, 9 (01): 107-110.

[46] Luo WuQiang, Liu Weimin, Huang jianzhong, Xiao Xin, Zhao WuXiao, Lin Quan. Clinical observation of dominant eye transition before and after LASIK surgery [J]. Guangxi Medical, 2010, 32 (12): 1475-1477.
[47] Wang YuLong. Research of dominant eye changes and effect on satisfaction after Laser In Situ Keratomileusis [D]. Huazhong University of Science and Technology, 2010.

[48] Hu YuKun, Gao XiaoWei, Li XiaoHong, Xu LiQun, Guo YunLin. Clinical observation of monovision with LASIK for correction of refractive errors with concomitant presbyopia [J]. International Journal of Ophthalmology, 2008 (05): 971-972.

[49] Anderson HA, Hentz G, Glasser A, Stuebing KK \& Manny RE. Minus-lens-stimulated accommodative amplitude decreases sigmoidally with age: a study of objectively measured accommodative amplitudes from age 3. Invest Ophthalmol Vis Sci 2008; 49: 2919-2926.

[50] Charman WN. Developments in the correction of presbyopia I: spectacle and contact lenses. Ophthalmic Physiol Opt 2014; 34: 8-29.

[51] Ravikumar S, Bradley A, Bharadwaj S \& Thibos LN. Expanding binocular depth of focus by combining monovision with diffractive bifocal intraocular lenses. J Cataract Refract Surg 2016; 42: 1288-1296.

[52] Legras R, Hornain V, Monot A \& Chateau N. Effect of induced anisometropia on binocular through-focus contrast sensitivity. Optom Vis Sci 2001; 78: 503-509.

[53] Xiao J, Jiang C. Visual outcomes of LASIK-induced monovision in myopic patients with presbyopia. Am J Ophthalmol 2011; 151: 557; author reply 557-8. DOI: 10.1016/j.ajo.2010.10.015

[54] Bennett ES. Contact lens correction of presbyopia. Clin Exp Optom 2008; 91: 265-278.

[55] Gauthier CA, Holden BA, Grant T \& Chong MS. Interest of presbyopes in contact lens correction and their success with monovision. Optom Vis Sci 1992; 69: 858-862.

[56] Gupta N, Naroo SA \& Wolffsohn JS. Visual comparison of multifocal contact lens to monovision. Optom Vis Sci 2009; 86: E98-E105.

[57] Stein HA. The management of presbyopia with contact lenses: a review. CLAO J 1990; 16: 33-38.

[58] Johannsdottir KR \& Stelmach LB. Monovision: a review of the scientific literature. Optom Vis Sci 2001; 78: 646-651. 